\title{
Non-invasive Hypoglycemia Monitoring System using Extreme Learning Machine for Type 1 diabetes
}

\author{
Sai Ho Ling , Phyo Phyo San, and Hung T. Nguyen
}

\begin{abstract}
Hypoglycemia (low blood glucose level) is a medical emergency and is a very common in type 1 diabetic persons and can occur at any age. It is the major limiting factor to maintain tight glycemic control. The deficiency in glucose counter-regulation may even lead to severe hypoglycaemia. It is always threatening to the well-being of patients with Type 1 diabetes mellitus (T1DM) since more severe hypoglycemia leads to seizures or loss of consciousness and the possible development of permanent brain dysfunction under certain circumstances . Because of that, an accurate continuing hypoglycemia monitoring system is a very important medical device for diabetic patients. Traditionally, different electrochemical glucose meters are adopted to measure the blood glucose. However, they are invasive, discontinuing and uncomfortable methods or painful for patients. In this paper, we proposed a noninvasive hypoglycemia monitoring system using the physiological parameters of electrocardiography (ECG) signal such as heart rate and corrected QT intervals. To enhance the detection accuracy of the hypoglycemia, a new emerging technology called extreme learning machine (ELM) is developed to recognize the presence of hypoglycemic episodes. ELM have both universal approximation and classification capabilities and provides efficient unified solutions to generalize feed-forward neural networks. A real clinical study of sixteen children with T1DM are given in this paper to illustrate the performance of ELM and successfully applied to a non-invasive hypoglycemia monitoring system. The natural occurrence of nocturnal hypoglycemic episodes are associated with increased heart rates $(p<0.06)$ and increased corrected QT intervals $(p<0.001)$. For comparison purpose, different computational intelligence technologies such as swarm based neural network, multiple regression based-fuzzy inference system, and fuzzy system were applied to this hypoglycemia monitoring system. By using the proposed ELM-based neural network (ELM-NN), the testing sensitivity (true positive) and specificity
\end{abstract}

The authors would like to thank Dr. Nejhdeh Ghevondian, and Assoc. Prof. Timothy Jones for their contribution. This works was supported by a grant from Juvenile Diabetes Research Foundation International.

Sai Ho Ling ${ }^{1}$, and Hung T. Nguyen are with the Centre for Health Technologies, Faculty of Engineering and IT, University of Technology Sydney, Ultimo, NSW, Australia. ${ }^{1}$ Steve. Ling@uts.edu.au; phone: +61 (2) 95142390

Phyo Phyo San is with the Institute for Infocomm Research, A* Star, Singapore. 
(true negative) for detection of hypoglycemia is better than the other intelligence methods with faster training time.

\section{INTRODUCTION}

Type 1 diabetes usually arises in children or young adults and is characterized by the inability to produce insulin. Although treatment regimens and types of insulin available have developed over time, individuals with type 1 diabetes are still required to self inject with insulin to control their blood glucose levels. Insulin doses must be adjusted according to carbohydrate intake and the degree of physical activity being undertaken.

For patients with type 1 diabetes, hypoglycemia (low blood glucose level) is one of the most common side effects and it is well know as a barrier for achieving and maintaining tight glycemic control. Individuals who experience frequent episodes of hypoglycemia and those who have been on insulin therapy for a long period of time may develop impaired awareness of hypoglycemia. Impaired hypoglycaemic awareness occurs in approximately 25\% of individuals with type 1 diabetes. Severe hypoglycemia may lead to physical and psychosocial morbidities such as brain damage, loss of consciousness, depression and low self-esteem [1]. It is also well known as death-in-bed syndrome [2].

Firstly, hypoglycemia is literally translated as a state produced by a lower level of blood glucose. It represents a significant hazard in patients with Type 1 diabetes mellitus (T1DM) which is a chronic medical condition that occurs when the pancreas produces very little or no insulin. In [3], another definition is given to hypoglycemia as the occurrence of a wide variety of symptoms in association with a plasma glucose concentration of $50 \mathrm{mg} / \mathrm{dl}$ or less. In clinical studies [4], the events of hypoglycemia are classified ranging from mild to severe level after reviewing the background of hypoglycemia. Most surveys revealed that the tighter the glycemic control in the younger patient, the greater frequency of both mild and severe hypoglycemia [5]. The level of blood glucose low enough to define hypoglycemia may be different for different people, in different circumstances, and for different purposes and occasionally has been a matter of controversy. Most healthy adults maintain fasting glucose levels above $3.9 \mathrm{mmol} / \mathrm{l}(70 \mathrm{mg} / \mathrm{dl})$, and develop symptoms of hypoglycemia when the glucose level falls below $3.0 \mathrm{mmol} / \mathrm{l}$ (55 $\mathrm{mg} / \mathrm{dl}$ ). In [6], it has been reported that the severe hypoglycaemic episodes are defined in those whose documented blood glucose levels is $2.8 \mathrm{mmol} / \mathrm{l}(50 \mathrm{mg} / \mathrm{dl})$ and the patient are advised to 
take necessary treatment. In this paper, blood glucose levels less than $3.33 \mathrm{mmol} / \mathrm{l}(60 \mathrm{mg} / \mathrm{dl})$ are considered as hypoglycemic episodes [6].

Several research works have been reported where patients with type 1 diabetes have been found dead in an undisturbed bed [2]. Since hypoglycemia can occur at any age due to many different conditions, early detection and prevention of hypoglycemia is more important for diabetes patients. To prevent or minimize hypoglycemic morbidities and mortalities, it is vital to return the blood glucose level to normal soon after a hypoglycemic event occurs. The patients' conditions are obviously need to know; when the event of hypoglycemia is initiated, and/or how the early warning is detected [7]. In other words, the need for hypoglycemia monitoring device is very important to provide an alarm alert to type 1 diabetes patients or carer when low blood glucose level is detected.

Real-time continuous glucose monitoring has the potential to overcome diabetic complications and increase the likelihood of patients with diabetes by maintaining optimal glucose level without symptomatic hypoglycemia. In clinical practice recommendations, it has also been suggested that continuous glucose monitoring is especially useful in patients with type 1 diabetes in order to detect hypoglycemia unawareness and/or frequent episodes of hypoglycemia. With ever improving advances in diabetes diagnostic technology, different blood glucose monitoring systems, invasive, minimally invasive and non-invasive are tested and introduced by the use of various techniques with the choice of sample region as described in Fig. 1.

Minimally invasive devices [8] or non-invasive devices [9] have been tested and introduced with the aid of current technologies such as reverse iontophoresis, suction effusion fluid and nearinfrared spectroscopy. But they suffer from limitations in terms of measurement inaccuracy, high susceptibility to artefact noise, considerable time delays for obtaining results, uncomfortableness and long-term reproducibility. Also, the cost of strip and the boredom of making repeated measurements become the great barriers for Type 1 diabetic patients who frequently need to monitor their BGL as well as episodes of hypoglycemia. Sometimes, the manual monitoring of blood glucose increases the risk of severe hypoglycemia due to their low efficiency in detecting frequent episodes of hypoglycemia. To overcome the limitations of the invasive devices, noninvasive continuing hypoglycemic monitoring systems have been developed.

The development of non-invasive continuous glucose monitoring systems is the only way for achieving painless control of blood glucose level and improving life quality of diabetes patients 
with better regulation of hypoglycemia episodes. To achieve it, hypoglycemia monitoring systems have been investigated by the use of physiological parameters that are stimulated by falling blood glucose levels. For instance, Teledyne Sleep Sentry [10] and Abbott Freestyle Navigator CGMS [11].

Recently, computational intelligence technologies were investigated for non-invasive hypoglycemia monitoring system. Based on the highly correlation between physiological parameters and hypoglycemia, a non-invasive hypoglycemia monitor was tested and introduced by Nguyen et al. [12] [13]. This system is employed by Bayesian neural network algorithm and the physiological parameters such as heart rate (HR), corrected QT interval (QTc) and skin impedance are used. The sensitivity of this device was found to be satisfactory, but the specificity need to be improved. More advanced neural network algorithms are still needed for obtaining better testing sensitivity and specificity. In addition, fuzzy estimator [14], fuzzy neural network estimator [15] were proposed for hypoglycemia detection systems. In these systems, the status of hypoglycemia was predicted through the changes in physiological parameters of the ECG signal. However, the membership functions which express the linguistic terms for fuzzy inference rules have to be defined. In practise, there is no formal approach for defining these rules and the optimization of these membership functions in terms of generalizing the data is also very important. Usually, tuning parameters of membership function is a time consuming task.

In [16] [17], an evolved fuzzy reasoning model was developed to recognize the presence of hypoglycemic episodes. The physiological parameters such as heart rate and corrected QT interval of ECG signal were continuously measured for early detection of hypoglycemic episodes in Type 1 diabetes mellitus (T1DM) patients. In this method, the fuzzy rules and fuzzy membership functions were optimized by hybrid particle swarm optimization with wavelet mutation. The results showed that the proposed algorithm performed well in terms of the sensitivity and specificity. However, more parameters were need to be optimized and a large computational time is need for training. Further experiment were conducted by the use of neural network based rule discovery system [18]. The development was based on 420 data sets which were collected from 16 T1DM patients by using the genetic algorithm. Though the obtained sensitivity and specificity are reasonable, the neural network used in the developed detection system might not be able to improve further because its slow learning speed and poor computational scalability.

To overcome the challenges faced by the mentioned state-of-the-art computational intelligence 
techniques, an emerging new technique named extreme learning machine (ELM) [19] has been brought to the considerable attention. In this paper, ELM is used to train a single-hidden layer feed-forward neural network (FFNN) and used to detect the status of hypoglycemia. Different from common understanding of learning algorithm for FFNN, its hidden node parameters are randomly generated and no tuning process is necessary. Besides, it is able to achieve the smallest training error as well as the smallest norm of output weights. Due to its significant features including faster learning speed and better generalization, ELM-based hypoglycemia monitoring system is investigated in this paper. To illustrate the performance of the proposed ELM based hypoglycemia monitoring system, a study of 16 T1DM children with 589 sampled data is given and found that the proposed method is successful in detecting nocturnal hypoglycemic episodes with faster learning time.

This paper is organized as follows: in Section II, details of the development of feed-forward neural network and extreme learning machine are introduced. To show the effectiveness of our proposed methods, the results of the detection of nocturnal hypoglycaemia episodes in T1DM are discussed in Section III and a conclusion is drawn in Section IV.

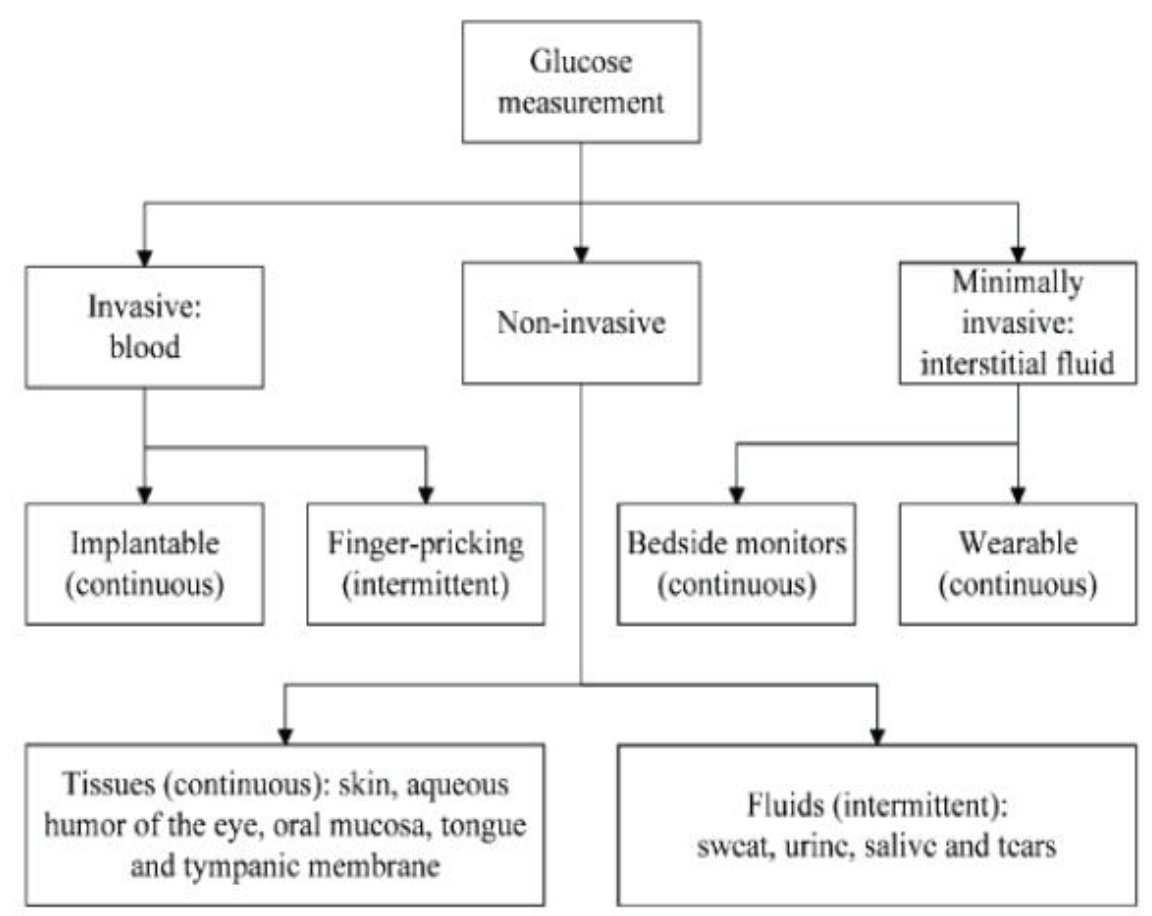

Fig. 1. Overview of technologies for blood glucose control: invasive, minimally invasive and non-invasive [Amaral2008] 


\section{METHODS}

To realize the detection of hypoglycemic episodes in T1DM patients, an ELM-based neural network is developed with four physiological parameters of ECG inputs and one output, as shown in Fig. 2. The four physiological parameters of ECG are heart rate (HR), corrected QT interval of ECG (QTc), change of heart rate ( $\Delta \mathrm{HR})$, and change of corrected QT interval of ECG ( $\Delta$ QTc), while output is the onset of hypoglycemia, +1 representing non-hypoglycemia and -1 representing hypoglycemia. The ELM-based neural network plays a important role in modeling the correlation between the four physiological parameters of ECG and the onset of hypoglycemia. For patient with type 1 diabetes, the possibility of hypoglycemia is mainly effected by prolongation of QT intervals (starting from the point of Q wave to at the end of $\mathrm{T}$ wave) and its correlation to heart rate. Not only $Q T_{c}$ interval prolongation has a significant impact on hypoglycemia, an increasing heart rate (HR) also may influence the onset of hypoglycemia.

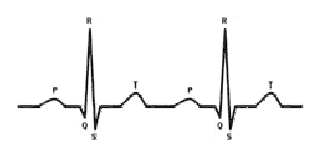

ECG Signals
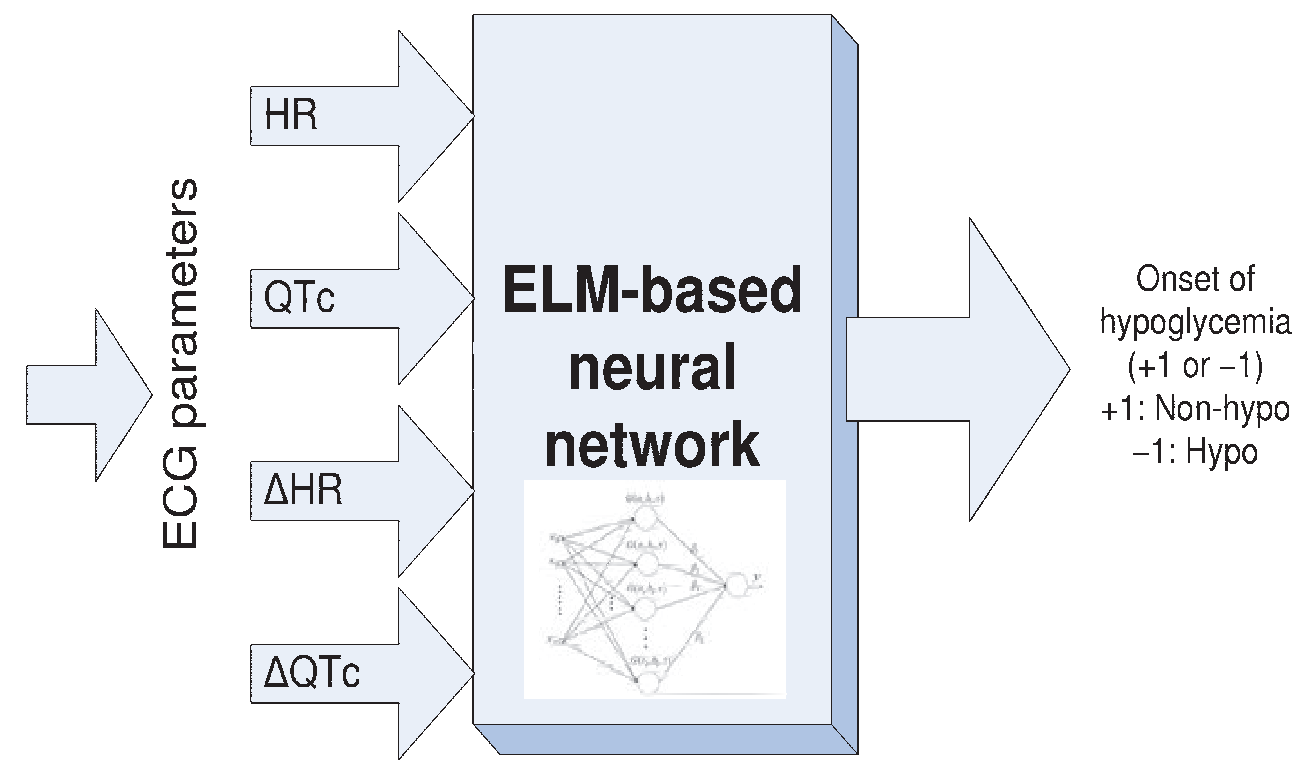

Fig. 2. ELM-based neural network for hypoglycemia monitoring system

\section{A. Electrocardiography (ECG)}

Hypoglycemia has long been known to affect the electrocardiogram (ECG) causing ST wave changes with lengthening of the QT interval and cardiac repolarization. These changes may increase the risk of cardiac arrhythmia; various abnormal heart rhythms, including ventricular 
tachycardia and atrial fibrillation [20]. These alternations are reflected by variations of ECG parameters, $\mathrm{Q}$ point, $\mathrm{R}$ peak and $\mathrm{T}$ wave peak including the commonly used $\mathrm{QT}$ interval (depolarization of the ventricles) which is the time taken from the start of the QRS complex to the end of the $\mathrm{T}$ wave (repolarization of ventricles) as presented in Fig. 3.

A number of studies have been carried out on the prolongation of QTc interval under both hyperinsulinemic hypoglycemia and spontaneous hypoglycemia and both conditions confirmed that QTc dispersion is increased in healthy individuals and in patients with type 1 [21].

The obtained results indicated that the degree of QTc lengthening during clamped hypoglycemia $(583 \mathrm{~ms})$ was greater compared to the euglycaemic control period (429 ms) with $p<0.001$. An example of QT measurement from one subject under euglycaemia and hypoglycemia was given in Fig. 4 in which the QT intervals were marked using an on screen cursor by two independent observers. As can be seen in Fig. 4, the increase in heart rate can cause a reduction in the QT interval and the QT interval is corrected for differences in heart rate by Bazett correction, dividing QT by the square root of RR, $(\mathrm{QTc}=\mathrm{QT} / \sqrt{R R})$ [22]. Not only QTc interval prolongation has a significant impact on hypoglycemia, but an increase in heart rate (HR) also may influence the onset of hypoglycemia.

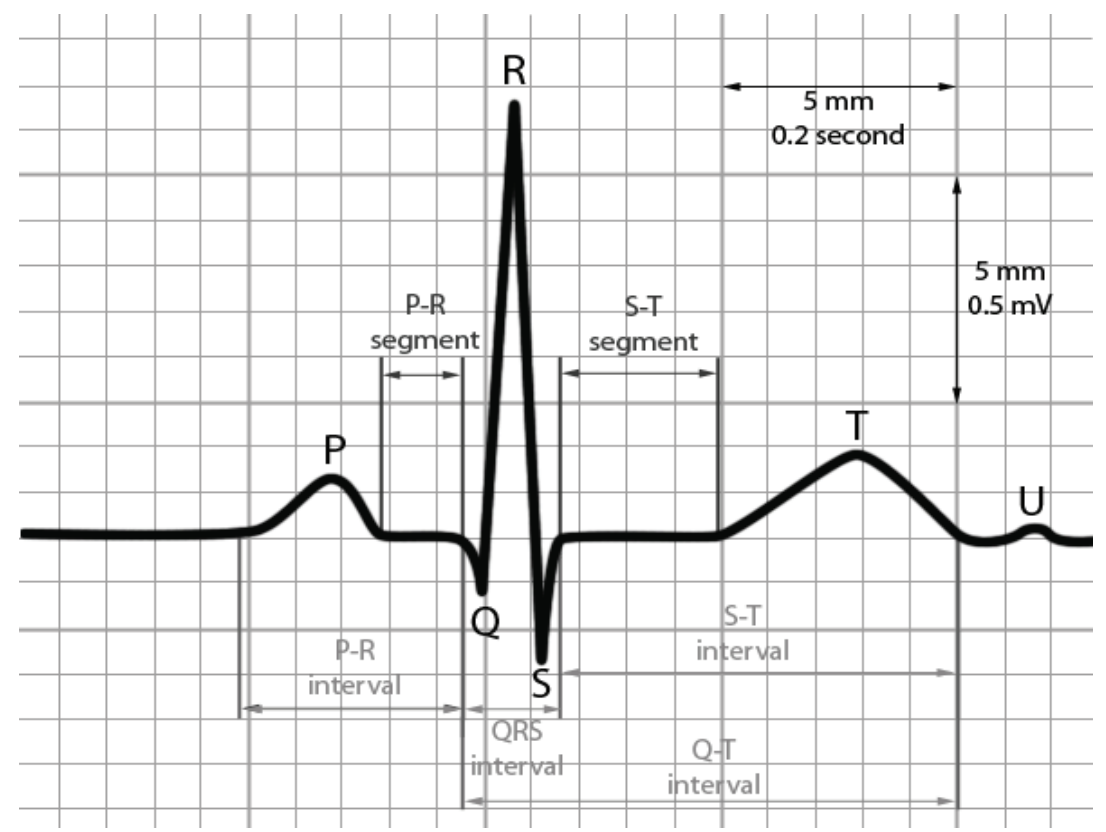

Fig. 3. ECG Signal 
A summary of the mean QTc lengthening during hypoglycemia [23], [24], [25], [26], [27], [28], [29] is shown in Table. I. It can be distantly seen that all the techniques produce a highly significant change of QTc for euglycemia and hypoglycemia conditions. Hence, the QTc prolongation during nocturnal hypoglycemia is of clinical interest importance because it may contribute to sudden death.

TABLE I

STUDIES OF CHANGES IN QTC DURING EUGLYCEMIC AND HYPOGLYCEMIC STUDIES

\begin{tabular}{|l|l|l|l|l|}
\hline \multirow{2}{*}{ Subjects } & \multicolumn{2}{|c|}{ Euglycemia } & \multicolumn{2}{c|}{ Hypoglycemia } \\
\cline { 2 - 5 } & QTc $(m s)$ & $\begin{array}{l}\Delta \text { QTc } \\
(m s)\end{array}$ & QTc $(m s)$ & $\begin{array}{l}\Delta \text { QTc } \\
(m s)\end{array}$ \\
\hline 8 T1DM and 7 Healthy [23] & 429 & 18 & 583 & 162 \\
10 Healthy [24] & 400 & 20 & 450 & 70 \\
17 Healthy [25] & 406 & 16 & 480 & 90 \\
8 T1DM [26] & 407 & 16 & 448 & 57 \\
17 Healthy [27] & 399 & 7 & 459 & 67 \\
16 T1DM and 8 Healthy [28] & 410 & 10 & 419 & 13 \\
18 Healthy [29] & 408 & 9 & 429 & 30 \\
\hline
\end{tabular}

Based on the above founding, we selected heart rate (HR), corrected QT interval (QTc), change of HR $(\Delta \mathrm{HR})$ and change of QTc $(\Delta \mathrm{QTc})$ as the inputs of the hypoglycemia monitoring system and the hypo detection will be done by ELM-based neural network.

\section{B. Extreme Learning Machine (ELM)-based neural network}

In this section, extreme learning machine (ELM) for the training of single hidden layer feedforward neural network (FFNN) in Fig. 5 is presented. Compared with the conventional neural network learning algorithms, it can achieve faster training performance and overcome the problem of over-fitting. The algorithm is based on the empirical risk minimization theory. Its learning process needs only for a single iteration and is able to avoid multiple iterations and local minimization. Because of better generalization ability, robustness, and controllability and faster learning process, it has been used in many real world applications [30]. For $N$ arbitrary distinct samples $\left(x_{i}, t_{i}\right) \in R^{d} \times R^{m}$, where $\mathbf{x}$ denotes the system inputs and $\mathbf{t}$ denotes the target 

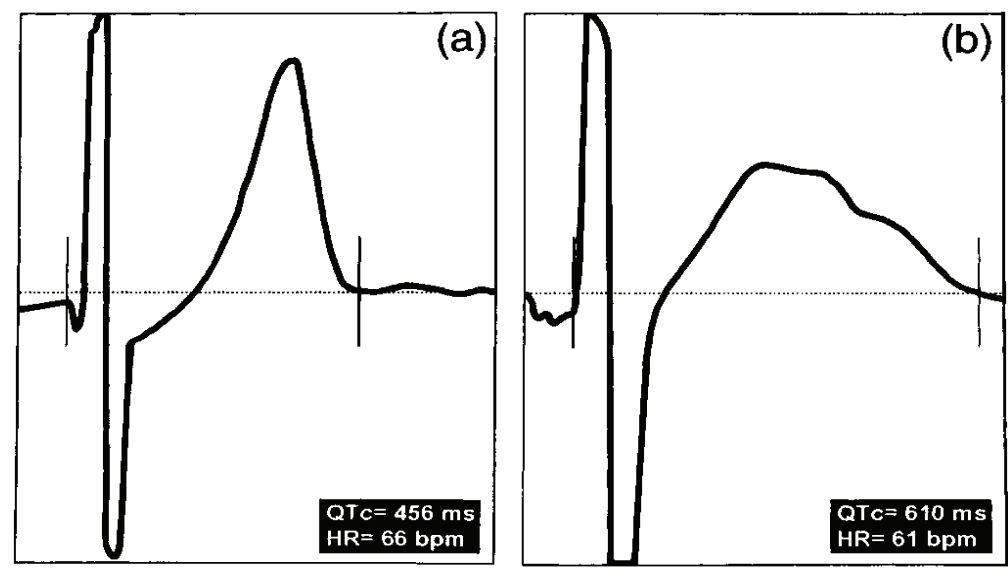

Fig. 4. Typical QT measurement with on screen cursor placement from one subject during euglycemia: (a) showing a clearly defined $\mathrm{T}$ wave (b) showing prolonged repolarization [23].

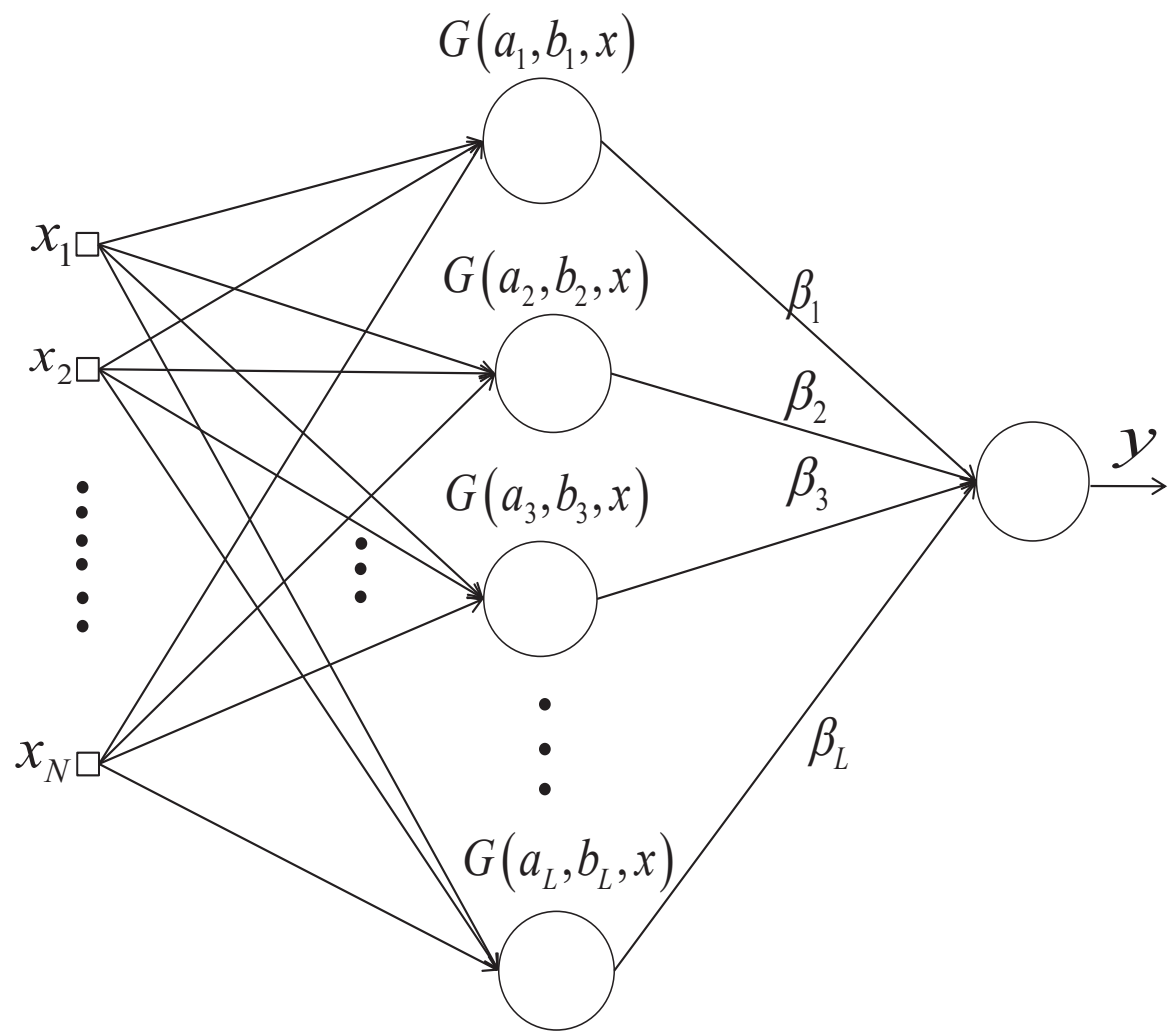

Fig. 5. Feed-forward neural network with single hidden layer 
output. The mathematically model of FFNN with $L$ hidden nodes can be written as:

$$
y_{j}=\sum_{i=1}^{L} \beta_{i} g_{i}\left(x_{j}\right)=\sum_{i=1}^{L} \beta_{i} G\left(a_{i}, b_{i}, x_{j}\right), \quad j=1, \ldots, N
$$

The FFNN can approximate these $N$ samples with zero error means that $\sum_{i=1}^{L}\left\|y_{j}-t_{j}\right\|=0$, i.e., there exist $\left(a_{i}, b_{i}\right)$ and $\beta_{i}$ such that $\sum_{i=1}^{L} \beta_{i} G\left(a_{i}, b_{i}, x_{j}\right)=t_{j}$, whereas $j=1, \ldots, N$. It can be expressed as:

$$
H \beta=T
$$

where

$$
\begin{gathered}
H=\left[\begin{array}{c}
h\left(x_{1}\right) \\
\vdots \\
h\left(x_{N}\right)
\end{array}\right]=\left[\begin{array}{ccc}
G\left(a_{1}, b_{1}, x_{1}\right) & \cdots & G\left(a_{1}, b_{1}, x_{1}\right) \\
\vdots & \cdots & \vdots \\
G\left(a_{1}, b_{1}, x_{1}\right) & \cdots & G\left(a_{1}, b_{1}, x_{1}\right)
\end{array}\right] \\
\beta=\left[\begin{array}{c}
\beta_{1}^{T} \\
\vdots \\
\beta_{L}^{T}
\end{array}\right]_{L \times m}, \quad T=\left[\begin{array}{c}
t_{1}^{T} \\
\vdots \\
t_{N}^{T}
\end{array}\right]_{N \times m}
\end{gathered}
$$

$H$ is called the hidden layer output matrix of the FFNN, the $i$ th column of $H$ is the $i$ th hidden node output with respect to inputs $x_{1}, x_{2}, \ldots, x_{N}, h(x)=G\left(a_{1}, b_{1}, x\right) \ldots G\left(a_{L}, b_{L}, x\right)$ is called as the hidden layer feature mapping. From the interpolation capability point of view, if the activation function $G$ is infinitely differentiable in any interval the hidden layer parameters can be randomly generated [19]. It means that the hidden layer output matrix $H$ in (3) is initialized with random weight $\left(a_{i}\right)$ and bias $\left(b_{i}\right)$ and calculate the output weight matrix $\beta$ from the linear equation in (2) by:

$$
\beta=H^{\dagger} T
$$

where $H^{\dagger}$ is the Moore-Penrose generalized inverse of the matrix H. To obtain $H^{\dagger}$, the orthogonal projection method can be efficiently used, whereas $H^{\dagger}=\left(H^{T} H\right)^{-1} H^{T}$ (or) $H^{\dagger}=H^{T}\left(H H^{T}\right)^{-1}$ if $H^{T} H$ and $H H^{T}$ are nonsingular. Substituting the output weight matrix in (1) to (5) and calculate the corresponding output of FFNN. The proposed hypoglycemia detection system, FFNN trained by ELM algorithm is given in detail as follows:

1) The hidden node parameters, $\left(a_{i}, b_{i}\right), i=1, \ldots, L$ are randomly generated. 
2) The hidden layer output matrix $H$ in (3) is calculated.

3) Obtain the output weight vector $\beta$ in (5) by $\beta=\left(H^{T} H\right)^{-1} H^{T} T$ (or) $\left(H H^{T}\right)^{-1} H^{T} T$.

4) The output of FFNN , $y$ is calculated by (1) and it is defined as positive when the status of hypoglycemia $h$ is positive which expressed as:

$$
h=\left\{\begin{array}{cc}
+1, & y \geq 0 \\
-1, & y<0 .
\end{array}\right.
$$

According to conventional neural network theories, hidden layer parameters, ie., weight and biases $\left(a_{i}\right.$ and $\left.b_{i}\right)$ and the output weights $\beta_{i}$ need to be tuned properly and searching for optimal parameters. Much efforts have been tried for obtaining better way of finding and adjusting hidden layer parameters $\left(a_{i}, b_{i}\right)$. In [31], incremental learning method which mainly focus and adjust on the newly added hidden nodes rather than adjusting all the hidden nodes parameters. In contrast, all the parameters of FFNN such as weight $\left(a_{i}\right)$ and biases $\left(b_{i}\right)$ and the output weight parameters $\left(\beta_{i}\right)$ need not be tuned and can be independent of training samples.

Similar to the conventional neural network, the numbers of hidden nodes are chosen by trial and error method until the optimal solution is obtained. However, there is a trade-off between too less number and too many numbers of hidden nodes because less number of node is insufficient to give the optimal solution and more numbers may unnecessarily increase the number of parameters to be tuned by ELM.

In essence, the ELM is not necessary to be tuned iteratively. Both the training error and the norm of weight can be minimized as long as the hidden layer feature mapping need to satisfy approximation condition [32]. In ELM, the hidden nodes can be randomly generated and the output weights vector $\beta_{i}$ is the only unknown parameters which can be resolved by ordinary leastsquare directly. Unlike the traditional computational intelligence techniques, ELM also provide better generalization performance at a much faster convergence rate.

\section{Clinical Study: Results And Discussions}

In this paper, clinical study with different computational intelligence technologies are presented and discussed. Sixteen children with T1DM (14.6 \pm 1.5 years) volunteered for the 10hour overnight hypoglycemia study at the Princess Margaret Hospital for Children in Perth, Western Australia. Each patient is monitored overnight for the natural occurrence of nocturnal 
hypoglycemia. Data were collected with approval from Women's and Children's Health Service, Department of Heath, Government of Western Australia, and with informed consent.

We measured the required physiological parameters, while the actual blood glucose levels were collected as reference using Yellow Spring Instruments. The main parameters used for the detection of hypoglycemia are the heart rate and corrected QT interval. The actual blood glucose profiles for 16 T1DM children are shown in Fig. 6. The duration approximately is $360-480$ mins. All data points of the patients are start recorded at night. In effect, it estimates the presence of hypoglycaemia at sample period $k_{s}$ based on the basic of the data at sampling period $k_{s}$ and the previous data at sampling period $k_{s}-1$. In general, the sampling period is 5-10 minutes and approximately 35-40 data points are used for each patient. The responses from 16 T1DM children exhibit significant changes during the hypoglycemia phase against the non-hypoglycemia phase. Normalization is used to reduce patient-to-patient variability and to enable group comparison by dividing the patient's heart rate and corrected QT interval by his/her corresponding values at time zero. The changes in HR and QTc were observed during the hypoglycemic event. In this group of patients, compared to the observed data during non-hypoglycemic episodes, their normalized heart rates $(\mathrm{HR})$ increased $(1.082 \pm 0.298$ vs. $1.033 \pm 0.242, p<0.06)$ and their normalized corrected QT intervals $\left(Q T_{c}\right)$ increased significantly $(1.060 \pm 0.0844$ vs. $1.031 \pm 0.086, p<0.001)$ during hypoglycemic episodes which was specified at $3.33 \mathrm{mmol} / 1$ [33]. These changes would last as long as hypoglycemic episodes persist. However, after the glucose level recovered, these changes in HR and QTc would be responded to normal within 5-10 minutes. For hypoglycemic episodes, the normalized mean of HR and QTc are decreased from 1.1423 to 1.0250 and 1.0656 to 1.0030 respectively.

Among sixteen patients, 12 patients were found to experience hypoglycemic episodes as their blood glycose level were less or equal than $3.33 \mathrm{mmol} / \mathrm{l}$, while 4 patients did not experience hypo events. Information for each patient in terms of number and duration of hypo events (BGL $\leq 3.33 \mathrm{mmol} / \mathrm{l})$ is tabulated in Table II. Patient numbers $1-8$ were used for training and patient number 9-16 were used for testing. The patients number are randomly chosen from these 16 patients. With these 16 patients, the number of sampled data points for training and testing are 320 and 269 respectively.

To measure the performance of hypoglycemia monitoring system for clinical studies, Sensi- 
tivity, $\xi$ and Specificity, $\eta$ are introduced [Altman1994]:

$$
\xi=\frac{N_{T P}}{N_{T P}+N_{F N}}, \quad \eta=\frac{N_{T N}}{N_{T N}+N_{F P}}
$$

where $N_{T P}$ is defined as number of true positive, $N_{F N}$ is number of false negative, $N_{F P}$ is number of false positive, and $N_{T N}$ is number of true negative. In clinical studies, sensitivity is more important than specificity because it identifies the actual hypoglycemic episodes in patients with T1DM. Thus, higher sensitivity are essential.

For comparison and analysis purposes, five methods are investigated to the hypoglycemia monitoring system as following:

1) Proposed ELM-based neural network(ELM-NN);

2) Particle swarm optimization based neural network(PSO-NN)[17];

3) Second order multiple regression fuzzy inference system (MR-FIS) [16];

4) Fuzzy inference system (FIS) [34];

5) Linear multiple regression (LMR).

For PSO-NN, a three-layer feed-forward neural network trained with hybrid particle swarm optimization with wavelet mutation is used. The settings of the PSO parameters can be found in [17]. For MR-FIS, a multiple regression with fuzzy inference system is applied to hypoglycemia application, genetic algorithm is used to optimize the fuzzy rules and the regression model. FIS and LMR are also applied to this application for comparison purpose. The number of membership function for each input (total 4 inputs) of FIS is five and the total number of fuzzy rules is 625 .

All of the methods are applied into the hypoglycemia monitoring system where the four inputs of the system are heart rate (HR), QT interval (QTc), $\Delta H R$ and $\Delta Q T c$ and one output to represent the onset of hypoglycemia. The testing results in term of sensitivity, specificity, gamma value are tabulated in Table III. Moreover, the training time for all methods are also shown in the same table to illustrate how fast of the proposed method compared with other methods. In this table, gamma $(\gamma)$ analysis is introduced in (7) to evaluate the actual performance of this biomedical application. As mentioned, the performance of sensitivity is more important, thus, the ratio of sensitivity and specificity is set to $60: 40(\theta=0.6$ for gamma analysis in (7) ).

$$
\gamma=\theta \xi+(1-\theta) \eta
$$




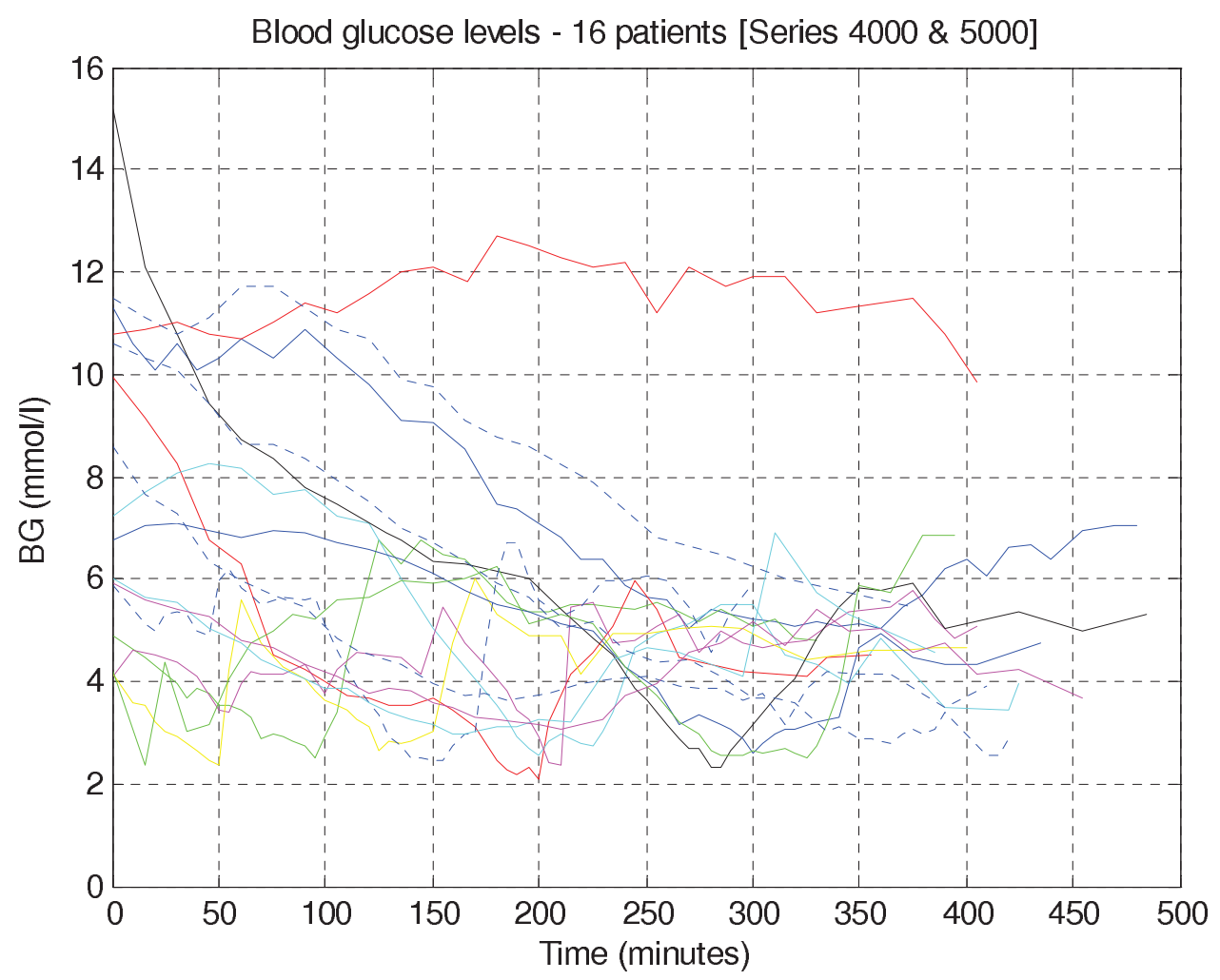

Fig. 6. Blood glucose level profiles for sixteen TIDM children

In Table III, we can seen that the testing sensitivity and specificity of the proposed ELM-NN achieve 78.00 and $60.00 \%$ which is better than MR-FIS (75.00\% and 50.23\% for testing sensitivity and specificity) and FIS (75.00\% and 51.04\% for testing sensitivity and specificity). In terms of gamma value, ELM-NN still performs better compared with other methods. To illustrate the efficiency of ELM, PSO-NN is used to compare. PSO-NN has same neural network structure of ELM-NN but trained with a hybrid PSO with wavelet mutation [17]. Given from the results, it can be seen that the performance of the ELM-NN is outperformed in terms of testing sensitivity, testing specificity, and gamma value. Besides, training time for all methods are given in same table. We can seen that the proposed ELM-NN gives a faster training time which is about 3 mins, while the other methods (PSO-NN, MR-FIS, and FIS) take around 30 to 275 mins. PSO-NN, trained by PSO and MR-FIS, and FIS are trained by genetic algorithm. Both evolutionary algorithm are time consuming and take longer time for training especially FIS involved 625 fuzzy rules need to be trained. LMR gives a very fast training time due to its simple modeling and only few tuning parameters, however, the testing sensitivity is unacceptable 
(51.78\% only). In Summary, the ELM-NN gives better results with faster training time.

\section{CONCLUSIONS}

In this paper, a non-invasive nocturnal hypoglycemia monitoring system for type 1 diabetes patients using extreme learning machine-based neural network model is developed. The results indicate that hypoglycemic episodes in type 1 diabetes mellitus children can be detected noninvasively and continuously effectively from the real-time physiological responses (heart rate and corrected QT interval). With the experimental result, which indicates that the proposed extreme learning machine based neural work performs better testing sensitivity and specificity with a faster training rate. As conclude, the testing performances of the proposed algorithm for detection of hypoglycemic episodes (sensitivity is $78.00 \%$ and specificity is $60.00 \%$ ) for T1DM are satisfactory.

There are two main limitations for the proposed hypoglycemia monitoring system should be considered. Firstly, all the data were recorded from children with type 1 diabetes and the age range is $14.6 \pm 1.5$ years. With these young people, there are no any heart problems to affect the heart rate and QT interval and there are no confounding conditions including in these data set. If the patients have heart problem where the detection performance accuracy may be affected. Secondly, we assume that the normal resting heart rate for all patients meet the international health association standard. 
TABLE II

INFORMATION FOR EACH PATIENT IN TERMS OF NUMBER AND DURATION OF HYPOGLYCEMIA EVENTS FOR HYPOGLYCEMIC EPISODES WHERE BGL $\leq 3.33 \mathrm{MMOL} / \mathrm{L}$.

\begin{tabular}{|c|c|c|c|c|c|}
\hline Patient number & Number of hypo events & Duration of events & Patient number & Number of hypo events & Duration of events \\
\hline 1 & 0 & $0 \min$ & 9 & 17 & 105 mins \\
\hline 2 & 8 & 40 mins & 10 & 0 & $0 \mathrm{~min}$ \\
\hline 3 & 7 & 45 mins & 11 & 0 & $0 \mathrm{~min}$ \\
\hline 4 & 10 & 50 mins & 12 & 9 & 45 mins \\
\hline 5 & 4 & 20 mins & 13 & 9 & 85 mins \\
\hline 6 & 14 & 75 mins & 14 & 13 & 70 mins \\
\hline 7 & 9 & 45 mins & 15 & 7 & 70 mins \\
\hline 8 & 11 & 55 mins & 16 & 0 & $0 \mathrm{~min}$ \\
\hline
\end{tabular}

TABLE III

TESting Results And TRAining time

\begin{tabular}{|l|c|c|c|c|}
\hline Algorithms & Sensitivity & Specificity & gamma value $(\gamma)$ & Training time \\
\hline ELM-NN & $78.00 \%$ & $60.00 \%$ & $70.80 \%$ & $3 \mathrm{mins}$ \\
\hline PSO-NN & $63.64 \%$ & $54.67 \%$ & $60.05 \%$ & $30 \mathrm{mins}$ \\
\hline MR-FIS & $75.00 \%$ & $50.23 \%$ & $65.09 \%$ & $40 \mathrm{mins}$ \\
\hline FIS & $75.00 \%$ & $51.64 \%$ & $66.55 \%$ & $275 \mathrm{mins}$ \\
\hline LMR & $51.78 \%$ & $51.64 \%$ & $51.72 \%$ & $1 \mathrm{~min}$ \\
\hline
\end{tabular}

\section{REFERENCES}

[1] L. G. Frederick and J. Zrebiec, "Detection of hypoglycemia by children with type 1 diabetes 6 to 11 years of age and their parents: a field study", Pediatric, vol. 121, no. 3, pp. e489-e495, 2008. 
[2] D. C. Klonoff, "The need for hypoglycemia detection and prevention in type 1 diabetes", Diabetes Technology and Therapeutics, vol. 3, no. 4, pp. 567-570, 2001.

[3] J. B. Field, "Hypoglycemia: definition, clinical presentations, classification, and laboratory tests", Diabetes Research Laboratory, vol. 18, no.1, pp. 27-43, 1989.

[4] A. D. Association, "Standards of medical care in diabetes", Diabetes Care, vol. 31, pp. 12-54, 2008.

[5] D. J. Becker and C. M. Ryan, "Hypoglycemia: A complication of diabetes therapy in children”, Trends in Endocrinology and Metabolism, vol. 11, no. 5, pp 198-202,2000.

[6] DCCT Research Group, "Adverse events and their association with treatment regimens in the diabetes control and complications trial",Diabetes Care, vol. 18, no. 11, pp. 1415V1427, 1995.

[7] A. Osareh and B. Shadgar, "Intrusion detection in computer networks based on machine learning algorithms", International Journal of Computer Science and Network Security, vol. 8, no. 11, pp. 15-23, 2008.

[8] J. Kimura and N. Ito and T. Kuriyama, "A novel blood glucose monitoring method", Chemical Sensors, vol. 87, no. 9, pp. 327-333, 1987.

[9] H. M. Heise and R. Marbach and T. Koschinsky, "Noninvasive blood glucose sensors based on near-infrared spectroscopy", Artificial Organs, vol. 18, no. 6, pp. 439-447, 1994.

[10] J. Castano and Y. Z. Wang and D. Chuang, "Blood glucose dependence of visual flicker threshold", Diabetes Technology and Therapeutics, vol. 2, no. 1, pp. 31-43, 2000.

[11] R. L. Weinstein, "Accuracy of the 5-day FreeStyle Navigator Continuous Glucose Monitoring System: comparison with frequent laboratory reference measurements", Diabetes Care, vol. 30, no. 5, pp. 1125-1130, 2007.

[12] H. T. Nguyen and N. Ghevondian and T. W. Jones, "Neural-network detection of hypoglycemic episodes in children with type 1 diabetes using physiological parameters", 28th Annual International Conference of the IEEE Engineering in Medicine and Biology Society, pp. 6053-6056, 2006.

[13] H. T. Nguyen and N. Ghevondian and S. T. Nguyen and T. W. Jones, "Detection of hypoglycemic episodes in children with type 1 diabetes using an optimal Bayesian neural network algorithm”, International Conference of IEEE Engineering in Medicine and Biology Society, pp. 3140-3143, 2007.

[14] N. Ghevondian and H. T. Nguyen, "Using fuzzy logic reasoning for monitoring hypoglycemia in diabetic patients", 19th Annual International Conference of the IEEE Engineering in Medicine and Biology Society, pp. 1108-1111, 1997.

[15] N. Ghevondian and H. T. Nguyen, "A novel fuzzy neural network estimator for predicting hypoglycaemia in insulin-induced subjects", 23rd Annual International Conference of the IEEE Engineering in Medicine and Biology Society, pp. 1657-1660, 2001.

[16] S. H. Ling and H. T. Nguyen, "Genetic-algorithm-based multiple regression with guzzy inference system for detection of nocturnal hypoglycemic episodes”, IEEE Transactions on Information Technology in Biomedicine, vol. 15, no. 2, pp. 308-315, 2011.

[17] S. H. Ling and H. T. Nguyen, "Natural occurrence of nocturnal hypoglycemia detection using hybrid particle swarm optimized fuzzy reasoning model”, Artificial Intelligence In Medicine, vol. 55, no. 3, pp. 177-184, 2012.

[18] K. Y. Chan, S. H. Ling, T. S. Dillon and H. T. Nguyen, "Diagnosis of hypoglycemic episodes using a neural network based rule discovery system", Expert Systems with Applications, vol. 38, no. 8, pp. 9799-9808, 2011.

[19] G. B. Huang, D. H.Wang, and Y. Lan, "Extreme learning machines: a survey", International journal of machine learning and cybernetics, vol. 2, pp. 107-122, 2011. 
[20] J. Benhorin, M. Merri and M. Alberti, ”Long QT syndrome: new electrocardiographic characteristics”, Circulation, vol. 82, no. 5, pp. 521-527, 1990.

[21] R. T. Robinson, N. D. Harris and R. H. Ireland, "Changes in cardiac repolarization during clinical episodes of nocturnal hypoglycaemia in adults with type 1 diabetes”, Diabetologia, vol. 47, pp. 312-315, 2004.

[22] A. D Association, "Standards of medical care in diabetes", Diabetes Care, vol 31, pp. 12-54, 2008.

[23] J. L. Marques, ”Altered ventricular repolarization during hypoglycaemia in patients with diabetes", Diabet Medicine, vol. 8, pp. 648-654, 1997.

[24] R. T. C. E. Robinson, N. D. Harris, and R. H. Ireland, "Mechanisms of abnormal cardiac repolarization during insulininduced hypoglycemia”, Diabetes, vol. 52, pp. 1469-1474, 2003.

[25] R. T. Robinson, N. D. Harris and R. H. Ireland, "Comparative effect of human soluble insulin and insulin aspart upon hypoglycaemia-induced alterations in cardiac repolarization”, Br J Clin Pharmacol, vol. 55, no. 3, pp. 246-251, 2003.

[26] S. P. Lee, N. D. Harris and R. T. Robinson, "Effect of atenolol on QTc interval lengthening during hypoglycaemia in type 1 diabetes", Diabetologia, vol. 48, no. 7, pp. 1269-1272, 2005.

[27] R. H. Ireland, R. T. Robinson and S. R. Heller, "Measurement of high resolution ECG QT interval during controlled euglycaemia and hypoglycaemia”, Physiological Measurement, vol. 21, no. 2, pp. 295-303, 2000.

[28] M. L. Koivikko, M. Karsikas, P. I. Salmela, "Effects of controlled hypoglycaemia on cardiac repolarisation in patients with type 1 diabetes", Diabetologia, vol. 51, no. 3, pp. 426-435, 2007.

[29] M. D. T. Laitinen and T. L. Laitinen, ”Electrocardiographic alterations during hyperinsulinemic hypoglycemia in healthy subjects", Annals of Noninvasive Electrocardiology, vol. 13, no. 2, pp. 97-105, 2008.

[30] G. B. Huang, Q. Y. Zhu, and C. K. Siew, "Extreme learning machine: theory and applications", Neurocomputing, vol. 70, pp. 489-501, 2006.

[31] A. R. Barron, "Universal approximation bounds for superpositions of a sigmoidal function", IEEE Transactions on Information Theory, vol. 39, no. 3, pp. 930-945, 1993.

[32] G. B. Huang, H. Zhou, X. Ding, and R. Zhang, "Extreme learning machine for regression and multiclass classification", IEEE Transactions Systems, Man, and Cybernetics, Part B, vol. 42, pp. 513-529, 2012.

[33] H. T. Nguyen, N. Ghevondian, and T. W. Jones, ”Detection of nocturnal hypoglycemic episodes (natual occurrence) in children with type 1 diabetes using an optimal bayesian neural network algorithm”, n 30th Annual International conference of the IEEE Engineering in Medicine and Biology Society, Canada, Aug 2008, pp. 1311-1314.

[34] S. H. Ling, Nuryani, H. T. Nguyen, "Evolved fuzzy reasoning model for hypoglycemic detection", in 32nd Annual International conference of the IEEE Engineering in Medicine and Biology Society, Argentina, Aug 2010, pp. 4662-4665. 